\title{
Ab initio treatment of electron correlations in polymers: lithium hydride chain and beryllium hydride polymer
}

\author{
Ayjamal Abdurahman, ${ }^{1}$ Alok Shukla, 2 , \\ ${ }^{1}$ Max-Planck-Institut für Physik komplexer Systeme, Nöthnitzer Str. 38, D-01187 Dresden, \\ Germany \\ ${ }^{2}$ Department of Physics and The Optical Sciences Center, University of Arizona, Tucson, AZ \\ 85721
}

\begin{abstract}
Correlated $a b$ initio electronic structure calculations are reported for the polymers lithium hydride chain $[\mathrm{LiH}]_{\infty}$ and beryllium hydride $\left[\mathrm{Be}_{2} \mathrm{H}_{4}\right]_{\infty}$. First, employing a Wannier-function-based approach, the systems are studied at the Hartree-Fock level, by considering chains, simulating the infinite polymers. Subsequently, for the model system $[\mathrm{LiH}]_{\infty}$, the correlation effects are computed by considering virtual excitations from the occupied HartreeFock Wannier functions of the infinite chain into the complementary space of localized unoccupied orbitals, employing a full-configuration-interaction scheme. For $\left[\mathrm{Be}_{2} \mathrm{H}_{4}\right]_{\infty}$, however, the electron correlation contributions to its ground state energy are calculated by considering finite clusters of increasing size modelling the system. Methods such as Møller-Plesset second-order perturbation theory and coupled-cluster singles, doubles and triples level of theory were employed. Equilibrium geometry, cohesive energy and polymerization energy are presented for both polymers, and the rapid convergence of electron correlation effects, when based upon a localized orbital scheme, is demonstrated.
\end{abstract}

\section{INTRODUCTION}

Polymers represent a class of one-dimensional infinite crystalline systems where ab initio Hartree-Fock (HF) self-consistent field (SCF) methods are well developedll. An available program package is CRYSTAL . However, in order to be able to calculate the structural and electronic properties of polymers with an accuracy that allows a meaningful comparison with experiment, it is usually necessary to include the effects of electron correlations into the theory. The most widely used approach here is density-functional theory (DFT). Despite its indisputable success in solid state physics and computational chemistry as a computationally cheap routine tool for large-scale investigations, DFT has the drawback that results depend highly on the chosen functional, and cannot be improved in a systematic way. Wavefunction-based quantum chemical ab initio techniques on the other hand are free from this flaw, and provide a large array of methods of different accuracy and computational cost. Thus it is desirable to extend their applicability to infinite systems such as polymers. 
Electron correlations are mostly a local effect and therefore localized molecular orbitals are preferable to the canonical HF solutions for the treatment of large molecules. 3 Similarly, in infinite systems (localized) Wannier functions provide a better starting point for an $a b$ initio treatment of electronic correlations than the (canonical) Bloch functions. Previous studies of polymers obtained the Wannier orbitals from an a posteriori localization of the Bloch functions according to a given prescription. During the last years, in our group a HF approach was developed which allows the direct determination of Wannier orbitals within the SCF process. 1 Various applications to one- and three-dimensional infinite systems proved the numerical equivalence of our Wannier-function-based HF approach to the conventional Bloch-function-based counterpart.

In this paper $\mathrm{HF}-\mathrm{SCF}$ calculations and subsequent correlation energy calculations are presented for the lithium hydride chain $[\mathrm{LiH}]_{\infty}$ and the beryllium hydride polymer $\left[\mathrm{Be}_{2} \mathrm{H}_{4}\right]_{\infty}$. As a simple, but due to its ionic character, nontrivial model polymer, the lithium hydride chain system has been previously dealt with in a number of studies.6. 6 . In the present contribution we extend our previous calculation 6 to a wave-function-based $a b$ initio study of electron correlation effects using a combination of the full configuration interaction (FCI) method and the the so-called incremental scheme. 911 The latter approach consists basically in an expansion of the total correlation energy per unit cell in terms of interactions of increasing complexity among the electrons assigned to localized orbitals (Wannier functions) comprising the polymer under consideration. The electron correlation energy increments needed to establish the total energy per unit cell are evaluated by considering virtual excitations from a small region of space in and around the reference cell, keeping the electrons of the rest of the crystal frozen at the Hartree-Fock (HF) level. The fast convergence of the incremental expansion allows to truncate it at relatively low order and thus to calculate the correlation energy of an infinite system without modelling it as a finite cluster. However, neither the FCI method nor the incremental approach based on polymer Wannier orbitals can at present be used for systems with a more complicated unit cell. Therefore, the second system investigated by us, the beryllium hydride polymer, was treated at the coupled-cluster (CC) and Møller-Plesset second-order perturbation (MP2) level of theory. Starting from the Wannier HF data the correlation corrections to the total energy per unit cell were derived from quantum chemical calculations of finite model systems using the MOLPRO molecular orbital ab initio program package.12 To our knowledge, this system was studied at the HF level two decades ago by Karpfen using the crystal orbital method, i.e., without including correlation effects 13 Recently, its monomer beryllium dihydride $\mathrm{BeH}_{2}$ has been well characterized theoretically using reliable ab initio and density functional theory methods.14.15

The remainder of the paper is organized as follows. In section II the applied methods are briefly described. The calculations and results are then presented in section [II. Finally, a summary is given in section $\mathbb{I V}$.

\section{APPLIED METHODS}

Section $\Pi \mathrm{A}$ gives brief outline for the theory within a restricted HF (RHF) framework. Sections $\amalg \mathrm{IB}$ and $\amalg \mathrm{IG}$, respectively, describe the incremental scheme and a simple approach, to compute electron correlation effects in polymers. 


\section{A. Wannier-orbital-based Hartree-Fock approach}

Our approach, described in more detail in previous publications. 6 is based upon the direct determination of the orthonormal Wannier-type (localized) orbitals for the polymer. Denoting by $\left|\alpha\left(\mathbf{R}_{\mathbf{j}}\right)\right\rangle$ the Wannier orbitals of a unit cell located at lattice vector $\mathbf{R}_{\mathbf{j}}$, the set $\left\{\left|\alpha\left(\mathbf{R}_{i}\right)\right\rangle ; \alpha=1, n_{c} ; j=1, N\right\}$ spans the occupied HF space. Here, $n_{c}$ is the number of orbitals per unit cell, and $N(\rightarrow \infty)$ is the total number of unit cells in the system. In our previous work we showed that one can obtain $n_{c}$ RHF Wannier functions, $\left\{|\alpha\rangle, \alpha=1, n_{c}\right\}$ occupied by $2 n_{c}$ electrons localized in the reference unit cell (denoted $\mathcal{C}$ ) by solving the equations 46

$$
\left(T+U+\sum_{\beta}\left(2 J_{\beta}-K_{\beta}\right)+\sum_{k \in \mathcal{N}} \sum_{\gamma} \lambda_{\gamma}^{k}\left|\gamma\left(\mathbf{R}_{k}\right)\right\rangle\left\langle\gamma\left(\mathbf{R}_{k}\right)\right|\right)|\alpha\rangle=\epsilon_{\alpha}|\alpha\rangle
$$

where $T$ represents the kinetic-energy operator, $U$ represents the interaction of the electrons of $\mathcal{C}$ with the nuclei of the whole of the crystal, while $J_{\beta}, K_{\beta}$, respectively, represent the Coulomb and exchange interactions felt by the electrons occupying the $\beta$-th Wannier function of $\mathcal{C}$, due to the rest of the electrons of the infinite system. The first three terms of Eq.(1) constitute the canonical Hartree-Fock operator, while the last term is a projection operator which makes the orbitals localized in $\mathcal{C}$ orthogonal to those localized in the unit cells in the immediate neighborhood of $\mathcal{C}$ by means of infinitely high shift parameters $\lambda_{\gamma}^{k}$ s. These neighborhood unit cells, whose origins are labeled by lattice vectors $\mathbf{R}_{k}$, are collectively referred to as $\mathcal{N}$. The projection operators along with the shift parameters play the role of a localizing potential in the Fock matrix, and once self-consistency has been achieved, the occupied eigenvectors of Eq.(1) are localized in $\mathcal{C}$, and are orthogonal to the orbitals of $\mathcal{N}$-thus making them Wannier functions $\mathbf{\theta}$. 6 . As far as the orthogonality of the orbitals of $\mathcal{C}$ to those contained in unit cells beyond $\mathcal{N}$ is concerned, it should be automatic for systems with a band gap once $\mathcal{N}$ has been chosen to be large enough. Based upon our past experience regarding a suitable choice of $\mathcal{N}, \mathbf{0}$ in the present calculation we included up to the third nearest-neighbor unit cells in $\mathcal{N}$. For the details concerning the computation

of various terms involving lattice sums $(U, J$, and $K$ ) involved in Eq. (11) for the case of polymers, we refer the reader to reference 6 .

\section{B. Incremental method}

Electron correlation effects in the ground states of a large number of three-dimensional ionic and covalent solids, 16 as well as polymers 17 have been studied with the incremental scheme. All these calculations used localized orbitals of finite clusters as a basis set for the correlation treatment. In the present work on the lithium hydride chain we use directly the Wannier-functions of the infinite system. A related study of the three-dimensional lithium hydride solid has been published elsewhere 19 .

The correlation energy per unit cell is expanded as

$$
E_{\text {corr }}=\sum_{i} \varepsilon_{i}+\sum_{<i j>} \Delta \varepsilon_{i j}+\sum_{<i j k>} \Delta \varepsilon_{i j k}+\ldots
$$


where the summation over $i$ involves Wannier functions located the reference cell, while those over $j$ and $k$ include all the Wannier functions of the crystal. The "one-body" increments $\varepsilon_{i}$ $=\Delta \varepsilon_{i}$ are computed by considering virtual excitations only from the $i$-th Wannier function, freezing the rest of the polymer at the HF level. The "two-body" increments $\Delta \varepsilon_{i j}$ are defined as $\Delta \varepsilon_{i j}=\varepsilon_{i j}-\left(\Delta \varepsilon_{i}+\Delta \varepsilon_{j}\right)$ where $\varepsilon_{i j}$ is the correlation energy of the system obtained by correlating two distinct Wannier functions $i$ and $j$. Thus $\Delta \varepsilon_{i j}$ represents the correlation contribution of electrons localized on two "bodies" $i$ and $j$. Higher-order increments are defined in an analogous way. Finally, summing up all increments, with the proper weight factors (according to their occurrence in the unit cell of the polymer), one obtains the exact correlation energy per unit cell of the infinite system. In order to get reliable results a size-extensive correlation method should be used, although non size-extensive schemes also may provide reasonable estimates if the incremental expansion is truncated at low order. In the present work for the lithium hydride chain we choose the strictly size-extensive full configuration interaction (FCI) method. As mentioned earlier, when computing the correlation contributions via Eq. (2), except for the orbitals involved (say orbitals $i$ and $j$ for the two-body increment $\Delta \epsilon_{i j}$ ), the rest of the occupied Wannier orbitals of the infinite solid are held frozen at the HF level. The region containing these frozen orbitals plays the role of the "environment" for the electrons involved in the correlated calculations, and its contribution can be absorbed in the so-called "environment potential" $U^{\text {env }}$ defined as

$$
U_{p q}^{\mathrm{env}}=\sum_{\alpha\left(\mathbf{R}_{j}\right) \in \mathcal{E}}\left(2\left\langle p \alpha\left(\mathbf{R}_{j}\right)\left|\frac{1}{r_{12}}\right| q \alpha\left(\mathbf{R}_{j}\right)\right\rangle-\left\langle p \alpha\left(\mathbf{R}_{j}\right)\left|\frac{1}{r_{12}}\right| \alpha\left(\mathbf{R}_{j}\right) q\right\rangle\right),
$$

where $\mathcal{E}$ represents the unit cells of the environment, $p$ and $q$ are two arbitrary basis functions, and the factor of two in the first term is due to the spin summation. The sum of Eq.(3) involves infinite lattice sum over the environment unit cells, and is computed by simply subtracting from the lattice summed $J$ and $K$ integrals (cf. Eq. (四)) obtained at the end of the HF iterations, the contributions corresponding to the orbitals being correlated. Once $U_{p q}^{\text {env }}$ has been computed, one is left with an effective Hamiltonian involving a finite number of electrons located in the region whose Wannier orbitals are being correlated. Physically speaking $U_{p q}^{\text {env }}$ represents the influence of the environment electrons on the electrons being correlated, explicitly. In the present calculations the $\mathrm{Li} 1 s^{2}$ core shell was also kept frozen, and its contribution was also included in $U_{p q}^{\mathrm{env}}$. The basis functions $p$ and $q$ were restricted to those of the reference cell and the adjacent cells up to the third-nearest neighbors.

The virtual orbitals used for computing the correlation effects were also localized. They were obtained by first orthogonalizing the basis set to the occupied space by using corresponding projection operators, as suggested by Pulay.20 Subsequently the basis functions are orthogonalized to each other using the symmetric-orthogonalization procedure, yielding a localized and orthonormal virtual orbital set.19 The number of virtual orbitals per unit cell considered for a specific increment corresponds to the number of basis functions per unit cell minus the number of occupied orbitals per unit cell. The virtual orbitals have been expanded in the same basis set as described above for $U_{p q}^{\mathrm{env}}$. 


\section{A simple approach}

In principle the total energy $E_{t o t}$ per $\left[\mathrm{Be}_{2} \mathrm{H}_{4}\right]$ unit cell of beryllium hydride may be obtained as the limit

$$
E=\lim _{n \rightarrow \infty} \frac{E\left(B e_{2 n+1} H_{4 n+2}\right)}{n}
$$

i.e., by performing calculations for increasingly long oligomers $\mathrm{H}\left(\mathrm{Be} \mathrm{H}_{2}\right)_{2 n} \mathrm{BeH}$. In order to reduce finite-size effects due to the termination of the oligomers by one beryllium and two hydrogen atoms saturating the dangling bonds of $\cdot\left(\mathrm{BeH}_{2}\right)_{2 n} \cdot$, one may consider instead

$$
E=\lim _{n \rightarrow \infty} \triangle E_{n}=\lim _{n \rightarrow \infty}\left[E\left(B e_{2 n+3} H_{4 n+6}\right)-E\left(B e_{2 n+1} H_{4 n+2}\right)\right]
$$

i.e., the energy change between subsequent oligomers differing by a single unit cell. Therefore, identical unit cells were used as building blocks for both oligomers, i.e., the geometrical optimization was restricted only to parameters relevant for the polymer beryllium hydride. Since the convergence of $\triangle E_{n}$ with respect to $\mathrm{n}$ is much faster for the correlation contributions than for the HF energy, and HF programs treating the infinite system are at hand (CRYSTAL, WANNIER), we use Eq. (5) only for the correlation energy per unit cell. This approach has previously been used successfully in calculations for trans-polyacetylene, 17 and some boron-nitrogen polymers.18

\section{CALCULATIONS AND RESULTS}

\section{A. $[L i H]_{\infty}$}

HF ground state calculations are a necessary prerequisite for the application of the incremental approach to electron correlation. We performed such calculations for a lithium hydride chain oriented along the $\mathrm{x}$-axis using the WANNIER code21. The reference cell contained hydrogen at the $(0,0,0)$ and lithium at the $(a / 2,0,0)$, where $a$ is lattice constant. We adopted the extended basis set optimized by Dovesi et al.22 First, all-electron Wannier HF calculations were performed at the different lattice constants in the range 2.8-4.0 $(\AA)$ and the total HF energy per unit cell for various lattice constants near the equilibrium was fitted to a cubic polynomial in order to derive the ground state HF equilibrium lattice constant and total energy. After determining the Wannier orbitals for each value of the lattice constant, the corresponding FCI calculations were performed by means of the incremental scheme. The expansion of the correlation energy per unit cell was restricted to one- and two-body increments, and included interactions up to third-nearest neighbor unit cells. Contributions from higher order increments as well as from interactions between more distant cells proved to be negligible. The equilibrium values for the FCI energy per unit cell and the lattice constant were determined as described for the HF results. The main contribution of $98.8 \%$ to the correlation energy per unit cell at the equilibrium geometry ( $E=-0.0307 a . u$.) comes from the one-body term. Two-body terms for first-, second- and third-nearest neighbors contribute with 1.15, 0.01 and $0.001 \%$, respectively. Our results are summarized in table 
口. It is quite obvious from table 1 that, as a function of distance, the two-body correlation effects converge very rapidly.

Since the Li basis set used here is suitable only for the ionic $\mathrm{LiH}$ molecule, we cannot get a good result for the atomic reference energy of the neutral $\mathrm{Li}$ atom (which is needed to determine the cohesive energy). Therefore, for this almost ideally ionic chain the cohesive energies both at the HF and the correlated level are obtained by subtracting the electron affinities (EA) and ionization potential (IP) from the dissociation energy calculated with respect to the ions $\mathrm{Li}^{+}$and $\mathrm{H}^{-}$. The HF values of EA and IP are determined using the finitedifference atomic HF program MCHF23. The experimental values of EA and IP were taken as the CI limit, i.e., disregarding the very small relativistic effects. For the polymerization energy we optimized the $\mathrm{Li}-\mathrm{H}$ distance for the ${ }^{1} \Sigma^{+}$ground state of the monomer at the $\mathrm{HF}$ and CI level. Our results are summarized in table [1]. It is clear from table II that, as expected, correlation effects contribute significantly to the cohesive energy. However, they do not make any significant contribution to the lattice constant of the system.

\section{B. $\left[\mathrm{Be}_{2} \mathrm{H}_{4}\right]_{\infty}$}

Beryllium hydride has attracted considerable interest as a rocket fuel on account of its high heat of combustion. It has also been considered as a moderator for nuclear reactors. From the previous studies 24 we also know that it is poisonous and difficult to prepare for experiment. Even though there is no or very little experimental information about the polymer, it has been studied theoretically using reliable ab initio methods at the HF level by Karpfen 13. In the present work we have studied this polymer at the HF and the correlated level. The Wannier-orbital-based HF-SCF approach, coupled-cluster (CC), and MøllerPlesset second--order perturbation (MP2) theory were employed to determine the equilibrium structures and total energies per unit cell. In our calculations the unit cell included two beryllium and four hydrogen atoms and has a perfect tetrahedral structure with all four Be$\mathrm{H}$ bond distances equal, i.e., there are two $\mathrm{HBeHBeH}$ planes that are perpendicular to each other, with the beryllium atoms in their crossings. In the cluster approximation the unit cell is terminated by one beryllium and two hydrogen atoms. In this structure the terminal beryllium atoms have trigonal coordination while all others are distorted tetrahedrons. First we optimized the structure of this polymer at the HF-SCF level using the CRYSTAL 2 program. The total HF energies obtained with the CRYSTAL program were then taken as an input for a re-optimization at the MP2, CCSD (CC singles and doubles) and CCSD(T) (CCSD with a perturbative estimate of triples) level. The correlation energy contributions at each geometry have been calculated with the MOLPRO molecular orbital ab initio program package 12 by using the simplified finite-cluster approach in which we put $n=3$ in Eq. (5). In this system the correlation energy converges rapidly with respect to cluster size, i.e., for $n=3$, one finds $\triangle E_{4}-\triangle E_{3} \approx 10^{-6}$ a.u.. We have optimized the beryllium-hydride bond length $\left(r_{B e H}\right)$ and the lattice constant $(\mathrm{a})$. We adopted polarized valence double-zeta $\left(6-31 G^{* *}\right)$ basis sets for beryllium and for hydrogen. The polarization functions consisted of a single p-type exponent of $0.75 \mathrm{Bohr}^{-2}$ on hydrogen and single d-type exponents of $0.4 \mathrm{Bohr}^{-2}$ on beryllium. In our HF calculations for polymers we optimized the most diffuse s-type exponent, which is less than 0.1 in the original $6-31 \mathrm{G}^{* *}$ basis set, and obtained 0.15 . A smaller value causes linear dependencies in the basis set when applied in the infinite system. 
We have also calculated the cohesive energy per unit cell at the HF and correlated level. The atomic HF-SCF, MP2, CCSD and CCSD(T) reference energies (Be: - 14.5668 a.u., -14.5928 a.u., -14.6131 a.u. and -14.6131 a.u.; H: -0.4982 a.u.) were obtained with the original $6-31 \mathrm{G}^{* *}$ basis sets. In addition to the cohesive energy, we have also calculated the polymerization energy. The geometry of the monomers was optimized at the SCF, MP2, CCSD, and CCSD $(\mathrm{T})$ levels of theory employing the MOLPRO program 12 . Our final results are summarized in table [II]. Due to the absence of experimental data or theoretical results at the correlated level, we compare our result only at the HF level. To the best of our knowledge, only Karpfen 13 has performed a geometry optimization for this polymer within an ab initio crystal Hartree-Fock approach and his results are also given in table III. Our beryllium-hydrogen bond length is in good agreement with the one obtained by Karpfen, but our HF energy is lower 0.05 a.u. than the value of Karpfen 13 . A possible reason is the use of $\mathrm{d}$ functions in our basis sets.

\section{SUMMARY}

In conclusion, given a well-localized basis set of Wannier orbitals size-extensive standard quantum chemical methods such as full configuration interaction, coupled-cluster or manybody perturbation theory can be applied to evaluate ground state properties of polymers. Rapid convergence of the incremental expansion of the correlation energy is obtained for ionic systems, e.g., the simple model of the lithium hydride chain. In beryllium hydride

polymer electron correlation accounts for $12-14 \%$ of the cohesive energy and $22-24 \%$ of the polymerization energy at all three levels of theory and reduces the lattice constant. In all the cases it was demonstrated that the use of localized orbitals leads to a rapid convergence of electron correlation effects, thus making it possible for one to compute the electron correlation effects of infinite systems. 


\section{REFERENCES}

* email: ayjamal@mpipks-dresden.mpg.de

$\dagger$ Present address: Department of Physics, Indian Institute of Technology, Powai, Mumbai 400 076, India

¥ Permanent address: Institut für Physikalische und Theoretische Chemie, Universität Bonn, Wegeler Str. 12, 53115 Bonn, Germany

${ }^{1}$ See, e.g., J. J. Ladik, Quantum Theory of Polymers as Solids. Plenum press, New York, NY (1988).

${ }^{2}$ R. Dovesi, V.R. Saunders, C. Roetti, M. Causa, N.M. Harrison, R. Orlando, E. Apra CRYSTAL95 user's manual. University of Turin, Italy (1996).

${ }^{3}$ C. Hampel and H. -J. Werner, J. Chem. Phys. 104, 6286 (1996), and references cited therein.

${ }^{4}$ A. Shukla, M. Dolg, H.Stoll and P. Fulde, Chem. Phys. Lett. 262, 213 (1996).

${ }^{5}$ A. Shukla, M. Dolg, P. Fulde, and H. Stoll, Phys. Rev. B, 57, 1471 (1998).

${ }^{6}$ A. Shukla, M. Dolg, and H. Stoll, Phys. Rev. B 58, 4325 (1998).

${ }^{7}$ H. Teramae, Theor. Chim. Acta 94, 311 (1996).

${ }^{8}$ D. Tunega, J. Noga, Theor. Chim. Acta 100, 78 (1998).

${ }^{9}$ H. Stoll, Phys. Rev. B 46, 6700 (1991).

${ }^{10}$ H. Stoll, J. Chem. Phys. 97, 8449 (1992).

${ }^{11}$ H. Stoll, Chem. Phys. Lett. 191, 548 (1992).

${ }^{12}$ H.-J. Werner and P. Knowles, MOLPRO, 1994, is a package of ab initio programs written by H.-J. Werner and P.J. Knowles, with contributions from J.Almlöf, R. D. Amos, A. Berning, C. Hampel, R. Lindh, W. Meyer, A. Nicklass, P. Palmieri, K.A. Peterson, R.M. Pitzer, H. Stoll, A.J. Stone, P.R. Taylor.

${ }_{13}^{13}$ A. Karpfen, Theor. Chim. Acta 50, 49 (1978).

${ }^{14}$ J. Hinze, O. Friedrich, and A. Sundermann, Mol. Phys, 96, 711 (1999).

${ }^{15}$ B. S. Jursic J. Mol. Struct. (Theochem) 467, 7 (1999).

${ }^{16}$ See, e.g., B. Paulus, P. Fulde and H. Stoll, Phys. Rev. B 54, 2556 (1996); K. Doll, M. Dolg, P. Fulde, and H. Stoll, Phys. Rev. B 55, 10282 (1997).

${ }^{17}$ M. Yu, S. Kalvoda, and M. Dolg, Chem, Phys 224, 121 (1997).

${ }^{18}$ A. Abdurahman, M. Albrecht, A. Shukla, and M. Dolg, J. Chem. Phys. 110, 8819 (1999).

${ }^{19}$ A. Shukla, M. Dolg, P. Fulde, and H. Stoll, Phys. Rev. B 60, 5211 (1999).

${ }^{20}$ P. Pulay, Chem. Phys. Lett. 100, 151 (1983).

${ }^{21}$ Computer program WANNIER, A. Shukla, M. Dolg, H. Stoll, and P. Fulde (unpublished).

${ }^{22}$ R. Dovesi, C. Ermondi, E. Ferrero, C. Pisani, and C. Roetti Phys. Rev. B 29, 3591 (1984).

${ }^{23} \mathrm{MCHF}$ atomic electronic structure code, C. Froese-Fischer, The Hartree-Fock Method for Atoms - A Numerical Approach, Wiley, New York, 1976.

${ }^{24}$ Ullmann's Encyclopedia of Industrial Chemistry, Fifth, Completely Revised Edition A13, 205 (1989). 


\section{TABLES}

TABLE I. Various increments to the correlation energy (in Hartrees) computed by the Wannier-function-based approach presented in this work. The results refer to lattice constant of 3.30 $(\AA)$. NN stands for nearest neighbors.

\begin{tabular}{lr}
\hline \hline Correlation & Energy \\
Increment & \\
\hline \hline one-body & -0.0303345 \\
two-body $(1 \mathrm{NN})$ & -0.0003538 \\
two-body $(2 \mathrm{NN})$ & -0.0000035 \\
two-body $(3 \mathrm{NN})$ & -0.0000003 \\
\hline \hline
\end{tabular}

TABLE II. Total energy $\mathrm{E}_{\text {tot }}$ (Hartree), cohesive energy $\triangle \mathrm{E}_{c o h}(\mathrm{eV})$, polymerization energy $\triangle \mathrm{E}_{\text {pol }}(\mathrm{eV})$ per unit cell and lattice constant a $(\AA)$ of the lithium hydride chain.

\begin{tabular}{lllll}
\hline \hline Method & $\mathrm{E}_{\text {tot }}$ & $\triangle \mathrm{E}_{\text {coh }}$ & $\triangle \mathrm{E}_{\text {pol }}$ & $\mathrm{a}$ \\
\hline \hline WANNIER SCF & -8.038047 & 3.8760 & 1.8067 & 3.3273 \\
CRYSTAL SCF & -8.038031 & 3.8759 & 1.8063 & 3.3274 \\
FCI & -8.068744 & 4.6545 & 1.4854 & 3.3300 \\
\hline \hline
\end{tabular}

TABLE III. Total energy $\mathrm{E}_{\text {tot }}$ (Hartree), cohesive energy $\triangle \mathrm{E}_{c o h}(\mathrm{eV})$, polymerization energy $\triangle \mathrm{E}_{\text {pol }}(\mathrm{eV})$ per unit $\mathrm{Be}_{2} \mathrm{H}_{4}$ and lattice constant a $(\AA)$, Be-H distance $\mathrm{h}(\AA)$ of beryllium hydride.

\begin{tabular}{llllll}
\hline \hline \hline Method & $\mathrm{E}_{\text {tot }}$ & $\triangle \mathrm{E}_{\text {coh }}$ & $\triangle \mathrm{E}_{\text {pol }}$ & $\mathrm{a}$ & $\mathrm{h}$ \\
\hline \hline CRYSTAL SCF & -31.6300 & 13.70 & 2.645 & 3.958 & 1.467 \\
$\mathrm{MP2}^{a}$ & -31.7608 & 15.85 & 3.445 & 3.958 & 1.456 \\
$\mathrm{CCSD}^{a}$ & -31.7908 & 15.56 & 3.402 & 3.969 & 1.457 \\
$\mathrm{CCSD}(\mathrm{T})^{a}$ & -31.7944 & 15.66 & 3.478 & 3.968 & 1.458 \\
Karpfen $^{b}$ & -31.5780 & - & - & 4.024 & 1.470 \\
\hline \hline
\end{tabular}

${ }^{a}$ correlation contributions added to CRYSTAL SCF energies.

${ }^{b}$ performed with $7,1 / 4$ basis sets considering third neighbor's interactions. 